\title{
Oil production optimization Solved by Piecewise linearization in a Branch \& Price framework
}

\author{
V. Gunnerud (NTNU),B. A. Foss (NTNU), \\ K. I. M. McKinnon (Edinburgh University),B. Nygreen (NTNU)
}

\begin{abstract}
This paper presents a method for optimizing oil production on large scale production networks such as the Troll west field in the North Sea. The method is based on piecewise linearization of all nonlinearities, and on decomposition of the full scale problem into smaller sub-problems. Column generation in a Branch \& Price framework is used to solve the decomposed problem. The methods differs from most Branch \& Price methods by branching only on continuous quantities and by solving the subproblems using commercial MILP software.

The method is applied to a realistic model of an oil field, the Troll oil and gas field at the Norwegian Continental Shelf, a petroleum asset with severe production optimization challenges due to rate dependent gas-coning wells. This study shows that the method is capable of solving instances of practical size to proven optimality.
\end{abstract}

Keywords: Oil production planning, oil rim reservoir, Mixed Integer Linear Programming, Column Generation, Branch \& Price. 


\section{Introduction}

Development of a gas and oil field requires planning on multiple horizons. On a long-term horizon, typically from one year and up to the field's lifetime, strategic reservoir planning is based on market conditions, field properties and the strategic considerations of the developing company. Decisions related to technology on this horizon will include; where to drill wells, whether to process the fluid onshore or offshore, and how to export the different products produced. The analyses and subsequent development plan seek to maximize the expected net present value of an asset by for instance maximizing oil and gas recovery. The article [Nygreen et al., 1998] discusses these issues.

On a medium-term horizon, typically from a few weeks to a year, the planner will seek specify an approximate operating policy so as to extract as much oil and gas from the reservoir as possible within the limits set by strategic decisions on investment and long-term operating policy. For example in the north west part of the Troll oil and gas field the extraction of gas is limited to ensure higher pressure in the reservoir for easier extraction of oil in the future.

This paper will focus on the real-time production optimization problem (RTPO), which has a short-term horizon of days to weeks. On this time scale the current state of the production system is known and the objective is to optimize the value of the production from the field subject to any current constraints on operation. Whenever the state of the system changes, for example due to breakdowns of equipment or new measurements, the RTPO has to be rerun, and so it is important that this can be done quickly.

Oil and gas production systems involve reservoirs, wells, gathering networks, and separators for splitting oil from gas and water, as well as systems for distributing the products. This paper models the optimization of the gathering network from the reservoirs to the inlet of the first separator (see Fig. 1). The boundary conditions are the pressure at the separator and the gas and water capacities of the separator. The data used in the study is based on the Norwegian Troll field.

The problem could be modelled as a mixed integer nonlinear program (MINLP). However the nonlinear equations which are needed to accurately describe the flows in pipes and wells are very complex, and including these in the model along with discrete variables for well connections would produce a model for which it would be unrealistic to expect to be able to guarantee global optimality. To overcome this problem we approximate the well and 


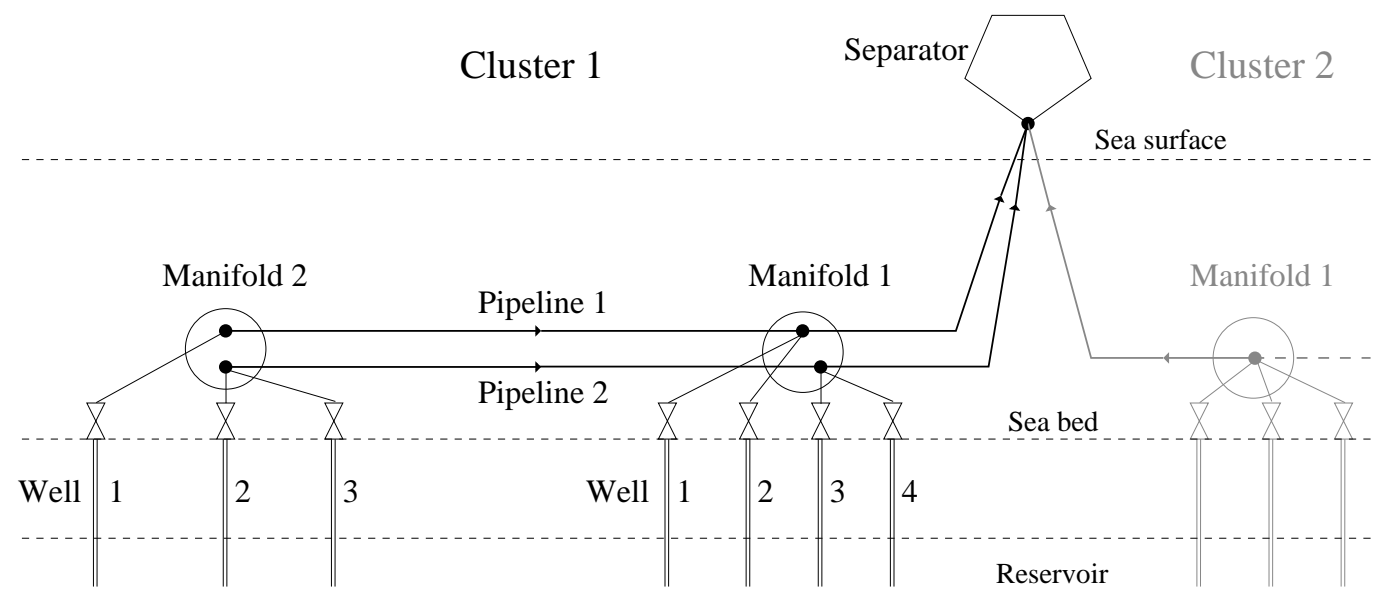

Figure 1: Cluster topology

pipe behaviour by a piecewise linearization using special ordered sets of type 2 (SOS2), which will yield a MILP approximation to the problem. This modular approach allows us to use results from any pipe and well simulators in our MILP optimization model.

The solution of this MILP would yield the global optimum. Unfortunately for many industrial problems it is not possible to solve this problem to global optimality using a standard commercial MILP solver: either the result is needed long before before the computation is complete or the computation fails due to lack of memory. To address this problem this paper develops a branch and price (B\&P) decomposition method which maintains the theoretical convergence properties of the standard approach but also converges much faster for large problems.

The remainder of this paper is organized as follows: Section 2 describes the real problem, Section 3 gives the MILP model, Section 4 describes the Branch \& Price and method, Section 5 give details of the implementation, Section 6 gives computational results and analysis, and Section 7 gives conclusions. 


\section{The Real Time Production Optimization Problem (RTPO)}

\subsection{Previous approaches and literature}

There are several commercial products in use today that attempt to solve the RTPO problem optimally, for example REO from [Weatherford, 2011], GAP from [Petroleum Experts, 2010], and MaxPro from [FMC Technologies, 2010]. These systems have realistic models of wells and pipes. They solve the optimization problem by a combination of linear and nonlinear techniques, but can not guarantee to find the global optima.

[Wang, 2003] provides a comprehensive overview of models and solution algorithms for different problems in the oil industry. He considers both linear and nonlinear formulations, with related techniques for solving them. A survey of the most common concepts and components of an oil production problem is presented in [Bieker et al., 2006], and other work on the subject is presented in [Saputelli et al., 2003]. Common to this literature, is a focus on the production chain from the reservoir to the inlet of the first separator, which is operated at a fixed pressure. Normally only a part of the problem is addressed or ad hoc rules are applied, which may lead to suboptimal solutions.

However, the following papers do address problems in the same category as in this work. [Bieker, 2007] solves a problem with simple network topology as a MILP, but without decomposition. [Kosmidis et al., 2005] deals with more flexible network topologies that allow routing of fluid streams from wells between different pipelines and to different separators. It solves the problems as an undecomposed MINLP using piecewise linearization of the wells' performance and outer approximation for the pipe characteristics, but because of non-convexity does not guarantee global optimality.

The following papers use a decomposition approach and model similar networks to the ones in this paper. [Foss et al., 2009] use Lagrangian relaxation using sub-gradient optimization to converge the prices and [Gunnerud and Foss, 2010] compare this with Dantzig-Wolfe decomposition. These methods differ only in how the prices are generated for the subproblems and both methods will give the same bound on the solution if continued to convergence. DantzigWolfe has a finite method of converging the upper bound and in the experiments it took fewer iterations to converge. Neither method is able to guaran- 
tee to find the global optima and on the problems in [Gunnerud and Foss, 2010] neither method dominated the other on this measure. [Gunnerud et al., 2010] and [Torgnes et al., 2012] show that the elapsed time to solve the DantzigWolfe method can be reduced by solving the subproblems in parallel. Also [Torgnes et al., 2012] extracts more solutions from each subproblem solve, which yields better feasible solutions but does not improve the bound.

When Dantzig-Wolfe and Lagrangian relaxation terminate they both give a bound on how sub-optimal the best solution found might be. However neither of them can guarantee to reach a pre-assigned tolerance. $\mathrm{B} \& \mathrm{P}$ on the other hand is able to give this guarantee. The first step of $\mathrm{B} \& \mathrm{P}$ is equivalent to Dantzig-Wolfe, but the method also contains a branching strategy to reduce the bound gap to any required tolerance. (In this paper all results are obtained to within $0.01 \%$ which is much lower than can be obtained without branching.) It is normal in $\mathrm{B} \& \mathrm{P}$ to branch on discrete variables, however the version in this paper branches on the continuous gas and water quantities.

The models and data used in this paper are more realistic than in the previous decomposition approaches - there are constraints on both gas an water, the effect of pipes outlet pressure is included and the data is derived from commercial simulators.

\subsection{Problem structure}

It is common in the offshore oil industry to have a production platform connected to several clusters each of which consists of a collection of wells and pipelines connecting the wells to the platform. An illustration of a typical cluster in an oil production system is showed in Figure 1. Different clusters may contain different numbers of pipelines, manifolds and wells. A pipeline consist of one or more pipes, each pipe either joining two manifolds or a manifold and the separator.

The flow of oil, gas and water from a well is a function of the wellhead pressure, and the relationship is known as the Well Performance Curve (WPC). The pressure drop through a pipe depends strongly on the flow of oil, gas and water through the pipe, and weakly on the inlet temperature and outlet pressure. The well flow and pipe pressure drop functions are highly nonlinear and accurate models of them requires complex thermodynamic and multi-phase flow calculations. We use commercial simulators for these calculations, and evaluate the functions at grids of points. Based on these values piecewise linear approximations are defined and included in the optimization 


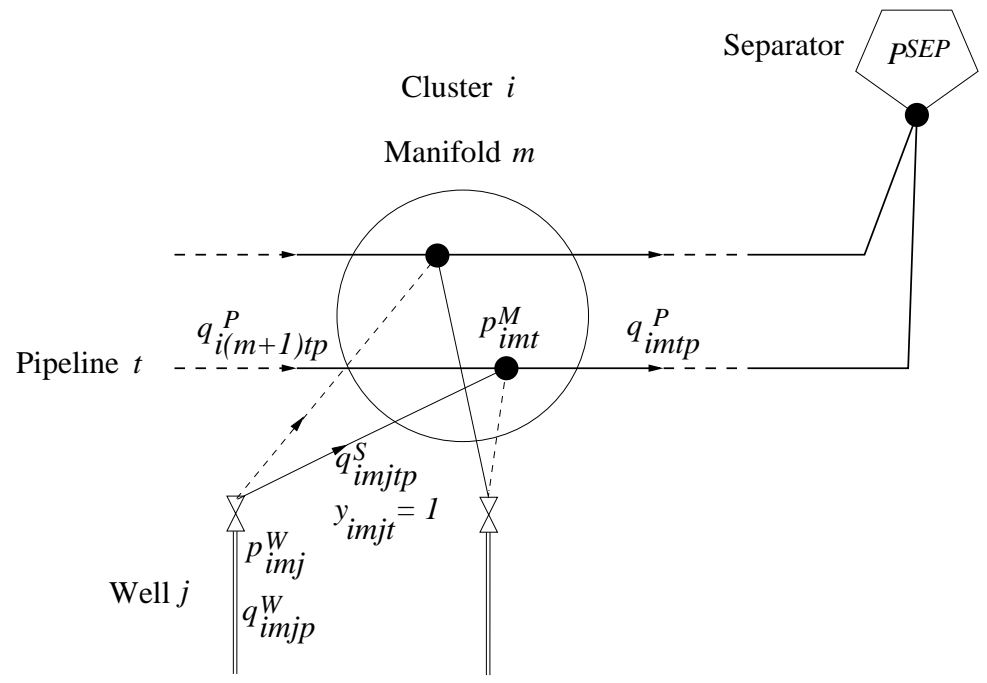

Figure 2: Illustration of manifold topology with variables

model. (See Section 3.)

Each well has a choke valve through which the well's output flows before entering the manifold and one of the pipelines. By operating this valve is it possible to increase the wellhead pressure and thereby reduce the flow. To prevent back flow into the well the model must ensure that whenever the choke is open to any extent the pressure in the manifold does not exceed the pressure in the wellhead. Furthermore a well cannot be connected to more than one pipeline, so when there is more than one pipeline in a manifold there is also a diverter valve which must be set to connect the well to exactly one of them. The fluid then flows through the pipeline to the production platform, which has a limited capacity for separating gas and water from the oil.

\section{Model formulation}

The following indexing conventions are used throughout the paper. Each cluster is identified by a single index $i$. Each manifold is identified by 2 indices $i m$, the index of the cluster in which it lies and its own index within the cluster. The index of the manifold is its number counting from the separator, which for convenience is treated as a manifold with index 0. Each 
well is identified by 3 indices $i m j, 2$ indices $i m$ to identify the manifold to which it is connected, and the index $j$ of the well within the group of wells connected to that manifold. Each pipeline is identified by 2 indices $i t$, the index of the cluster $i$ where it lies and an index $t$ of the pipeline within the cluster. Each pipe is identified by 3 indices $i m t$, the indexes it of the pipeline of which it is a part and the index $m$ of the manifold at its inlet end.

All the symbols used in the model are defined in Tables 1 to 5 All variables are non-negative, and the main ones are illustrated in Figure 2.

$i \quad$ - Cluster

Table 1: Indices

$m$ - Manifold when $m>0$, and separator when $m=0$

$j \quad-$ Well

$t$ - Pipeline

$p \quad$ - Phase (gas, oil and water)

$k \quad-$ Breakpoint index for the piecewise linearization of the well model

$n_{p} \quad-\quad$ Breakpoint index for the piecewise linearization of the pressure drop in pipes dependent on phase $\mathrm{p}$ ( $n_{g}$ for gas, $n_{o}$ for oil, $n_{w}$ water)

$\mathcal{I} \quad$ - Set of clusters

Table 2: Sets

$\mathcal{M}_{i} \quad$ - Set of manifolds $1 . . M_{i}$ in cluster $i$

$\mathcal{J}_{\text {im }} \quad$ - Set of wells connected to manifold $m$ in cluster $i$

$\mathcal{T}_{i} \quad-\quad$ Set of pipelines in cluster $i$

$\mathcal{P} \quad$ - Set of phases ( $g$ for gas, $o$ for oil, $w$ for water)

$\mathcal{P}^{C} \quad$ - Set of capacity constrained phases

$\mathcal{K}_{i m j} \quad$ - Set of indices of breakpoints for the piecewise linearization of the well performance curve (WPC) for well $i m j$

$\mathcal{N}_{i m p}$ - Set of indices of breakpoints for the phase $p$ coordinate in the piecewise linearization of the pressure drop in pipes imt (same for all piplines $t \in \mathcal{T}_{i}$ )

\section{Objective function}


Table 3: Parameters

$C_{p}^{T} \quad-\quad$ Capacity of phase $p$ in the first stage separator

$P^{S E P} \quad-$ Pressure at first-stage separator at platform $\left(=p_{i 0 t}^{M}\right)$

$P_{i m t n_{g} n_{o} n_{w}}^{D} \quad-\quad$ Pressure drop in pipe $i m t$ corresponding to interpolation indices $n_{g}, n_{o}$ and $n_{w}$ in the pipe pressure drop piecewise linearization

$P_{i m j}^{M A X} \quad-\quad$ Maximum difference between the wellhead pressure and the pressure inside the manifold

$P_{i m j k}^{W} \quad$ - Wellhead pressure of well $i m j$ for interpolation index $k$ in the piecewise linearization of the well performance curve (WPC)

$Q_{i m t p n_{p}}^{P} \quad-\quad$ Flow of phase $p$ in pipe $i m t$ interpolation index $n_{p}$.

$Q_{i m j p}^{W M A X} \quad-$ Maximum flow rate of phase $p$ from well $i m j$

$Q_{i m j p k}^{W} \quad-\quad$ Flow rate of phase $p$ from well $i m j$ corresponding to interpolation index $k$ in the piecewise linearization of the well performance curve (WPC)

$M_{i} \quad-\quad$ Number of manifolds in cluster $i$

The objective is to maximize the total oil flow from all clusters.

$$
\max z=\sum_{i \in \mathcal{I}} q_{i o}^{C}
$$

\section{Constraints}

The constraints can be segmented into the following groups:

Capacity constraints (2) are the only constraints that connect the clusters. They state that the sum of gas rates and water rates from all clusters must be less than the separator's gas handling capacity and water handling capacity respectively.

$$
\sum_{i \in \mathcal{I}} q_{i p}^{C} \leq C_{p}^{T} \quad \forall p \in \mathcal{P}^{C}
$$

Apart from these capacity constraints, all other constraints are defined locally for each cluster $i \in \mathcal{I}$. For the sake of simplicity this indexing definition will be omitted from the rest of the formulation.

The total flow from cluster $i$ is the sum of flows from the pipes entering 
Table 4: General Variables

$p_{i m t}^{M} \quad$ - Pressure in pipeline it in manifold or separator $m$

$p_{i m t}^{D} \quad$ - Pressure drop in pipe $i m t$ at the reference outlet pressure

$p_{i m j}^{W} \quad$ - Wellhead pressure in well $i m j$

$q_{i p}^{C} \quad-$ Total flow of phase $p$ from cluster $i$

$q_{i m t p}^{P} \quad$ - Flow rate of phase $p$ in pipe $i m t$

$q_{i m j p}^{W} \quad$ - Flow rate of phase $p$ from well $i m j$

$q_{i m j t p}^{S}$ - Flow rate of phase $p$ from well $i m j$ into pipeline $t$

$t_{i m t}^{T} \quad$ - Temperature at inlet of pipe $i m t$

$y_{i m j t} \quad$ Equals 1 if well $i m j$ is routed to pipeline $t, 0$ otherwise

Table 5: Interpolation Variables

$\gamma_{i m j k} \quad-\quad$ Weighting variable associated with interpolation index $k$ in the piecewise linearization of the WPC for well $i m j$

$\lambda_{i m t n_{g} n_{o} n_{w}}$ - Weighting variable associated with the interpolation indices $n_{g}, n_{o}, n_{w}$ in the piecewise linearization of the pressure drop in pipe $i m t$

$\eta_{i m t p n_{p}} \quad$ - Sum of all $\lambda_{i m t n_{g} n_{o} n_{w}}$ for a fixed value of $n_{p}$. Used in SOS2 sets for coordinate $p$ in the piecewise linearization of the pressure drop in pipe $i m t$

the separator.

$$
\sum_{t \in \mathcal{T}_{i}} q_{i m t p}^{P}=q_{i p}^{C} \quad \forall m=1, p \in \mathcal{P}
$$

The well model: The output from a well depends on the properties of the reservoir and the wellbore and is characterised by the Well Performance Curve (WPC). This specifies the output of each phase as a function of wellhead pressure. Our model uses the same piecewise linear approximation of the WPC as in [Gunnerud and Foss, 2010]. Let $P_{i m j k}^{W}$ be the $k^{t h}$ pressure breakpoint for well $i m j$ and let $Q_{i m j p k}^{W}$ be the corresponding flow of phase $p$. The piecewise linear approximations are shown in (4)-(7)

$$
\begin{array}{cc}
p_{i m j}^{W}=\sum_{k \in \mathcal{K}_{i m j}} P_{i m j k}^{W} \gamma_{i m j k} & \forall m \in \mathcal{M}_{i}, j \in \mathcal{J}_{i m} \\
q_{i m j p}^{W}=\sum_{k \in \mathcal{K}_{i m j}} Q_{i m j p k}^{W} \gamma_{i m j k} & \forall m \in \mathcal{M}_{i}, j \in \mathcal{J}_{i m}, p \in \mathcal{P}
\end{array}
$$




$$
\begin{array}{cc}
\sum_{k \in \mathcal{K}_{i m j}} \gamma_{i m j k}=1 & \forall m \in \mathcal{M}_{i}, j \in \mathcal{J}_{i m} \\
\gamma_{i m j k} \text { is SOS2 for } k & \forall m \in \mathcal{M}_{i}, j \in \mathcal{J}_{i m}
\end{array}
$$

Network logic constraints: The following inequality ensures that well $i m j$ is connected to at most one pipeline:

$$
\sum_{t \in \mathcal{T}_{i}} y_{i m j t} \leq 1 \quad \forall m \in \mathcal{M}_{i}, j \in \mathcal{J}_{i m}
$$

\section{Well and pipe pressure and flow linkage constraints}

$$
\begin{aligned}
\sum_{t \in \mathcal{T}_{i}} q_{i m j t p}^{S}=q_{i m j p}^{W} & \forall m \in \mathcal{M}_{i}, j \in \mathcal{J}_{i m}, p \in \mathcal{P} \\
q_{i m j t p}^{S} \leq Q_{i m j p}^{M A X} y_{i m j t} & \forall m \in \mathcal{M}_{i}, j \in \mathcal{J}_{i m}, t \in \mathcal{T}_{i}, p \in \mathcal{P}(10) \\
q_{i m t p}^{P}=\sum_{j \in \mathcal{J}_{i m}} q_{i m j t p}^{S}+q_{i(m+1) t p}^{P} & \forall 1 \leq m<M_{i}, t \in \mathcal{T}_{i}, p \in \mathcal{P} \\
q_{i m t p}^{P}=\sum_{j \in \mathcal{J}_{i m}} q_{i m j t p}^{S} & \forall m=M_{i}, t \in \mathcal{T}_{i}, p \in \mathcal{P} \\
p_{i m t}^{M} \leq p_{i m j}^{W}+P_{i m j}^{M A X}\left(1-y_{i m j t}\right) & \forall m \in \mathcal{M}_{i}, j \in \mathcal{J}_{i m}, t \in \mathcal{T}_{i}
\end{aligned}
$$

Constraint (9) states that the flow from a well has to be equal to the flow into the pipelines. Constraints (11) and (12) define the flow balance between wells and pipes at each manifold, (12) for the furthest out manifolds and (11) the others. When the connection between well $i m j$ and pipe imt is open, then $y_{i m j t}=1$ so (10) does not constrain the flow from the well to the pipe and (13) constrains the pressure in the manifold to be no higher than the wellhead pressure. When the connection is closed, then $y_{i m j t}=0$ and (10) ensures that there is no flow from the well into that pipeline, and (13) is relaxed so that there is no link between the wellhead and pipeline pressure.

Pipe Model: The pressure drop $F_{i m t}^{P}\left(q_{g}, q_{o}, q_{w}, p, t\right)$ between the inlet and outlet ends of pipe imt can be expressed as a function of the flows of gas $q_{g}$, oil $q_{o}$ and water $q_{w}$ and the inlet temperature $t$ and outlet pressure $p$. Over the range where they can vary, the variation of pressure drop with $t$ is not significant and the variation with $p$ is small. We therefore make the 
following approximation

$$
\begin{aligned}
F_{i m t}^{P}\left(q_{g}, q_{o}, q_{w}, p, t\right) & \simeq F_{i m t}^{P}\left(q_{g}, q_{o}, q_{w}, P_{i m t}^{R E F}, T_{i m t}^{R E F}\right)+\alpha_{i m t}\left(p-P_{i m t}^{R E F}\right) \\
\text { where } \alpha_{i m t} & =\frac{\partial F_{i m t}^{P}}{\partial p}\left(Q_{i m t g}^{R E F}, Q_{i m t o}^{R E F}, Q_{i m t w}^{R E F}, P_{i m t}^{R E F}, T_{i m t}^{R E F}\right)
\end{aligned}
$$

where the REF quantities are fixed and chosen to be in the middle of the expected range for the corresponding variables. $F_{i m t}^{P}\left(q_{g}, q_{o}, q_{w}, P_{i m t}^{R E F}, T_{i m t}^{R E F}\right)$ is a nonlinear function of the 3 variables $q_{g}, q_{o}$ and $q_{w}$ and we approximate it by a piecewise linear function in the same way as in [Gunnerud and Foss, 2010]. The model in that paper does not account for the variation of pressure drop with outlet pressure and is the special case of the model in this paper where all $\alpha_{i m t}=0$. The pressure drop variable for pipe $i m t$ at the reference outlet pressure $P_{i m t}^{R E F}$ is $p_{i m t}^{D}$, so in the model the equation for the approximate pressure drop is

$$
p_{i m t}^{M}-p_{i(m-1) t}^{M}=p_{i m t}^{D}+\alpha_{i m t}\left(p_{i(m-1) t}^{M}-P_{i m t}^{R E F}\right) \quad \forall m \in \mathcal{M}_{i}, t \in \mathcal{T}_{i}
$$

The piecewise linear function for $p_{i m t}^{D}$ is constructed as follows. Given the pipe's reference pressure $P_{i m t}^{R E F}$ and temperature $T_{i m t}^{R E F}$ the simulator is used to calculate

$$
P_{i m t n_{g} n_{o} n_{w}}=F_{i m t}^{P}\left(Q_{i m t g n_{g}}^{P}, Q_{i m t o n_{o}}^{P}, Q_{i m t w n_{w}}^{P}, P_{i m t}^{R E F}, T_{i m t}^{R E F}\right)
$$

for all breakpoints, where $Q_{i m t p n_{p}}^{P}$ is the flow of phase $p$ at the breakpoint with index $n_{p}$. Using this breakpoint data the piecewise linear function is now defined by (15) to (21) below.

$$
\begin{aligned}
p_{i m t}^{D}= & \sum_{n_{g} \in \mathcal{N}_{i m g}} \sum_{n_{o} \in \mathcal{N}_{i m o}} \sum_{n_{w} \in \mathcal{N}_{i m w}} P_{i m t n_{g} n_{o} n_{w}}^{D} \lambda_{i m t n_{g} n_{o} n_{w}} \quad \forall m \in \mathcal{M}_{i}, t \in \mathcal{T}_{i} \\
q_{i m t p}^{P}= & \sum_{n_{g} \in \mathcal{N}_{i m g}} \sum_{n_{o} \in \mathcal{N}_{i m o}} \sum_{n_{w} \in \mathcal{N}_{i m w}} Q_{i m t p n_{p}}^{P} \lambda_{i m t n_{g} n_{o} n_{w}} \forall m \in \mathcal{M}_{i}, t \in \mathcal{T}_{i}, p \in \mathcal{P} \\
& \sum_{n_{g} \in \mathcal{N}_{i m g}} \sum_{n_{o} \in \mathcal{N}_{i m o}} \sum_{n_{w} \in \mathcal{N}_{i m w}} \lambda_{i m t n_{g} n_{o} n_{w}}=1 \quad \forall m \in \mathcal{M}_{i}, l \in \mathcal{T}_{i}
\end{aligned}
$$

In addition we require that all non-zero $\lambda_{i m t n_{g} n_{o} n_{w}}$ weights are at vertices of a cube of neighbouring breakpoints. This condition can be enforced by 
$3 \mathrm{SOS} 2 \mathrm{~s}$, one for each of the gas, oil and water dimensions and defined as follows

$$
\begin{aligned}
& \eta_{i m t g n_{g}}=\sum_{n_{o} \in \mathcal{N}_{i m o}} \sum_{n_{w} \in \mathcal{N}_{i m w}} \lambda_{i m t n_{g} n_{o} n_{w}} \quad \forall m \in \mathcal{M}_{i}, t \in \mathcal{T}_{i}, n_{g} \in \mathcal{N}_{i m g} \\
& \eta_{\text {imtono }}=\sum_{n_{g} \in \mathcal{N}_{\text {img }}} \sum_{n_{w} \in \mathcal{N}_{\text {imw }}} \lambda_{i m t n_{g} n_{o} n_{w}} \quad \forall m \in \mathcal{M}_{i}, t \in \mathcal{T}_{i}, n_{o} \in \mathcal{N}_{\text {imo }} \\
& \eta_{\text {imtwn }}=\sum_{n_{g} \in \mathcal{N}_{\text {img }}} \sum_{n_{o} \in \mathcal{N}_{\text {imo }}} \lambda_{i m t n_{g} n_{o} n_{w}} \quad \forall m \in \mathcal{M}_{i}, t \in \mathcal{T}_{i}, n_{w} \in \mathcal{N}_{i m w} \\
& \eta_{\text {imtpn }_{p}} \text { is SOS2 for } n_{p} \quad \forall m \in \mathcal{M}_{i}, t \in \mathcal{T}_{i}, p \in \mathcal{P}
\end{aligned}
$$

It should be mentioned that only 4 neighboring $\lambda_{i m t n_{g} n_{o} n_{w}}$ are needed to enclose the solution, so 8 neighboring $\lambda_{i m t n_{g} n_{o} n_{w}}$ will therefore result in several degrees of freedom. These could be removed by including SOS2 sets on the diagonal as described in [Williams, 2005], however, as this adds complexity, we compensate instead by using more breakpoints.

Fixed separator pressure: To facilitate a compact formulation, the separator is defined as $m=0$. The pressure $P^{S E P}$ at the separator is fixed and is equal to the outlet pressure $p_{i 0 t}^{M}$ of all pipes $i 0 t$ connected to it, so

$$
p_{i 0 t}^{M}=P^{S E P} \quad t \in \mathcal{T}_{i}
$$

For pipe i1t, i.e. the one connected to the separator, we take the reference outlet pressure to be $P_{i 1 t}^{R E F}=P^{S E P}$. It follows that when $m=1$ the last term in (14) is zero.

\section{Branch \& Price (B\&P)}

In formulation (1) - (22) it is only the objective (1) and the constraints (2) that involve variables from more than one cluster. Further, each of these expressions involves only a sum of these variables, so by removing these constraints and instead dealing with them by pricing, the problem decomposes into independent subproblems, one for each cluster and each subproblem is itself a MILP. A common approach for problems of this form is Lagrangian relaxation, however in this paper we use branch \& price $(\mathrm{B} \& \mathrm{P})$. B\&P has the advantages of Lagrangian relaxation, and can in addition guarantee to find the global optimum. 


\subsection{Branch \& Price overview}

In $\mathrm{B} \& \mathrm{P}$ there is a master problem which constructs an optimal solution by selecting feasible solutions (or modes of operation) of subproblems and combining them optimally. The master problem contains constraints for the common resources (gas and water separation capacity in our case). Each column in the master problem corresponds to a mode of operation of a subproblem and its coefficients in the resource constraint rows are the total use of that resource by the subproblem mode. The master problem is solved by branch and bound and the problem at each node of the branch and bound tree is solved by column generation, [Desrosiers and Lübbecke, 2005].Column generation is used because there is an infinite number of possible subproblem modes so it is not possible to generate the master problem explicitly. The master problem at a B\&P node is solved iteratively. Each iteration starts by solving a Restricted Master Problem (RMP), which consists of a finite subset of the possible master problems columns. Then the subproblems are solved using the dual values for the common constraints to find the column with the most attractive reduced cost. If there is no attractive column the master problem at that $\mathrm{B} \& \mathrm{P}$ node is solved, otherwise a new column is added to the master and the iterations continue.

The solution of the master problem at a $\mathrm{B} \& \mathrm{P}$ node will consist for each subproblem of either a single mode of operation or a convex combination of modes. In most nodes there will be some subproblem with convex combination of modes that is not physically possible. To get round this problem and generate feasible solutions we also solve the RMP as an integer problem to select exactly one operating mode per subproblem.

In most $\mathrm{B} \& \mathrm{P}$ applications the branching is done on a discrete subproblem structure, though in some routing problems branching is done also on one continuous quantity, usually time. In this paper all the branching is done on the continuous capacity usages.

Also the subproblems in B\&P usually have a structure which can be solved effectively by dynamic programming, see [Irnish and Desaulniers, 2005] and [Amr and de Carvalho, 2005]. However, the subproblems presented in this paper have a more general structure, so we solve them using a commercial MILP solver, which is efficient and makes the implementation simpler. 


\subsection{Restricted master problem}

Let $\mu_{i s}$ be a variable representing the weighting within the RMP of operating mode $s$ of cluster $i$, and let $Q_{i s p}^{M O D E}$ be the total production of phase $p$ in this mode. Also let $\mathcal{S}_{i}$ be the set of existing modes for cluster $i$ which satisfy the cluster's gas and water production constraints at the current B\&P node. Then the RMP is as follows:

$$
\max \sum_{i \in \mathcal{I}} \sum_{s \in \mathcal{S}_{i}} Q_{i s o}^{M O D E} \mu_{i s}
$$

subject to

$$
\begin{aligned}
\sum_{i \in \mathcal{I}} \sum_{s \in \mathcal{S}_{i}} Q_{i s p}^{M O D E} \mu_{i s} & \leq C_{p}^{T} \quad \forall p \in \mathcal{P}^{C} \\
\sum_{s \in \mathcal{S}_{i}} \mu_{i s} & =1 \quad \forall i \in \mathcal{I} \\
\mu_{i s} & \geq 0 \quad \forall i \in \mathcal{I}, s \in \mathcal{S}_{i}
\end{aligned}
$$

Expression (23) is the objective function, (24) represents the constrained common resources, and (25) and (26) allows a convex combination of all solutions found for a given subproblem.

When this problem is solved, it produces a primal solution, $\mu_{i s}$, and a dual solution. If we denote the dual variables for (24) by $\pi_{p}^{C A P}$ and for (25) by $\pi_{i}^{C O N V E X}$, the reduced cost, $\bar{c}_{i s}$, for $\mu_{i s}$ can be expressed as:

$$
\bar{c}_{i s}=Q_{i s o}^{M O D E}-\sum_{p \in \mathcal{P}^{c}} \pi_{p}^{C A P} Q_{i s p}^{M O D E}-\pi_{i}^{C O N V E X} \quad \forall i \in \mathcal{I}, s \in \mathcal{S}_{i}
$$

\subsection{Structure of the master problem solution}

The RMP is an LP with one equality convexity constraint for each subproblem and $\left|\mathcal{P}^{C}\right|$ common inequality constraints. If we add slack variables for the inequalities, then since there is one basic variable per row, the problem will have $\left|\mathcal{P}^{C}\right|$ more basic variables than clusters. Hence the number of basic variables among the $\mu_{i s}$ variables is the number of clusters + the number of nonbasic slack variables, which is at most $\left|\mathcal{P}^{C}\right|$. Since the convexity constraints have positive RHS there must be at least one non zero $\mu_{i s}$ in each cluster, and this must be basic. In addition to these basic variables there can 
be up to $\left|\mathcal{P}^{C}\right|$ other basic variables among the $\mu_{i s}$. For the case in this paper when $\left|\mathcal{P}^{C}\right|=2$, there can be either 2 clusters with 2 basic variables each, or 1 cluster with 3 basic variables, or 1 cluster with 2 basic variables, or no clusters with more than one basic variable. Also since all nonbasic variables are 0 , if a cluster has a single basic variable, then this must be integer with value 1 . If the problem is non-degenerate, then clusters with more than one basic variable will be fractional, however this is not necessary the case if the problem is degenerate. Hence for our problems where $\left|\mathcal{P}^{C}\right|=2$ one of the following cases must occur: 2 clusters with 2 fractional values in each, 1 cluster with 2 or 3 fractional values, or 0 clusters with fractional values. It follows that except in cases of degeneracy or non-binding common capacities, the RMP solution will contain fractional weights.

\subsection{Subproblem}

If we want to improve the continuous solution of the RMP, we need to find a feasible mode of operation for at least one of the clusters which gives a positive reduced cost according to (27). If such a production mode exist, it can be found by solving separately for each cluster, $i$, the MILP subproblem

$$
z_{i}=\max q_{i o}^{C}-\sum_{p \in \mathcal{P}^{C}} \pi_{p}^{C A P} q_{i p}^{C}-\pi_{i}^{C O N V E X}
$$

subject to $(3)-(22)$ and

$$
Q_{i p}^{M I N} \leq q_{i p}^{C} \leq Q_{i p}^{M A X} \quad \forall p \in \mathcal{P}^{C}
$$

where $Q_{i p}^{M I N}$ and $Q_{i p}^{M A X}$ are the lower and upper bounds on $q_{i p}^{C}$ in the current $\mathrm{B} \& \mathrm{P}$ node. (Constraint (29) will be discussed later in relation to the branching.)

As mentioned earlier, these MILP subproblems will be solved by a commercial MILP solver that is able to treat SOS2 automatically. Whenever an optimal subproblem objective, $z_{i}$, is positive, we create a new column for the master, with index $s$ say, with entries $Q_{i s o}^{M O D E}$ in the objective row, $Q_{i s g}^{M O D E}$ and $Q_{i s w}^{M O D E}$ in the gas and water rows respectively, and a 1 in the convexity row for cluster $i$. Here $Q_{i s p}^{M O D E}$ for each phase $p$ is the optimal value of $q_{i p}^{C}$ in the solution for cluster $i$. Finally $s$ is added to set $S_{i}$.

If no column with a positive reduced cost is found for any of the clusters, then the current solution is the optimal continuous solution to the current node of the $\mathrm{B} \& \mathrm{P}$ tree. 


\subsection{Finding feasible solutions}

We noted in Section 4.3 that except in cases of degeneracy, the RMP solution will contain fractional weights. If any modes in a cluster that have fractional weights have a different set of nonzero binary or SOS2 variables, then the convex combination will not be feasible.

In order to find an integer feasible solution we solve the RMP (23) - (26) as a pure binary problem with (26) replaced by

$$
\mu_{i s} \in\{0,1\} \quad \forall i \in \mathcal{I}, s \in \mathcal{S}_{i}
$$

The set $\mathcal{S}_{i}$ here consists of all previously generated operating modes, not just those from the current B\&P node. This step is done after the solution of each $\mathrm{B} \& \mathrm{P}$ node. In the following, the IP version of the RMP is denoted RMP-IP while the LP version is denoted RMP.

\subsection{Branching strategy}

The lower bound on the optimal solution is given by the best RMP-IP solution, and the upper bound is given by highest optimal RMP objective value among all active nodes in the B\&P tree. To close this gap we need to create new modes of operation for the different clusters so that better IP solutions can be created, and we need to branch to tighten the LP upper bounds by splitting nodes.

In $\mathrm{B} \& \mathrm{P}$ it is normal to branch on the original discrete variables, see [Barnhart et al., 1998]. Our problem contains two types of discrete variables, the binary variables in (8), and the SOS2 variables in (7) and (21). There are very many of these variables and so it is likely that using them for branching will result in a very large number of branches. The column generation procedure however is time consuming, so only a small number of master B\&P nodes can be solved in a reasonable amount of time, and so this approach is unlikely to be successful.

In this paper, instead of branching on the original integer and SOS variables, we branch on the continuous gas and water flow variables from each cluster by imposing bounds on the available capacities. Columns which conflict with these new bounds are removed from the child nodes.

Our aim in choosing the branching variable $q_{i p}^{C}$ is to maximize the distance between the solution at the parent and the closer of the solutions at its children. This should keep the branch and bound tree balanced and avoid 
generating new production modes that are close to existing modes. We use the following method:

First identify all $\mu_{i s}$ with fractional values. (There must be at least one otherwise the node would be feasible and it would not have been branched on.) As noted in Section 4.3 the only cases that can occur are two clusters with 2 fractional variables each, or one cluster with 2 or 3 fractional variables. In the first case we need to select a cluster and a phase and in the other cases only a phase. The following rules are used in all cases:

Let the interpolated production of phase $p$ (gas or water) in the RMP solution of subproblem $i$ be denoted $Q_{i p}^{A V}$. For each subproblem $i$ such that $\mu_{i s}$ is fractional for some mode $s$, calculate

$$
D_{i s p}=\left|Q_{i s p}^{M O D E}-Q_{i p}^{A V}\right| \quad \forall p \in \mathcal{P}^{C}, \forall s \in \mathcal{S}_{i}, 0<\mu_{i s}<1
$$

( $D_{i s p}$ is the absolute difference between the RMP solution and a column used in the interpolation.) Then for each cluster $i$ with a fractional $\mu_{i s}$ and each phase $p$ find the second largest value of $D_{i s p}$ among the 2 or 3 modes $s$ with fractional $\mu_{i s}$ and denote this by $D_{i p}^{*}$. Then find the cluster $i^{*}$ and phase $p^{*}$ which give the largest relative values of these $D_{i p}^{*}$, i.e.

$$
i^{*}, p^{*}=\arg \max _{i, p} \frac{D_{i p}^{*}}{Q_{i p}^{A V}}
$$

Then branch on cluster $i^{*}$ and phase $p^{*}$. Finally set the branching value to $Q_{i p}^{A V}$, i.e. enforce the bound $q_{i p}^{C} \leq Q_{i p}^{A V}$ in the lower branch and $q_{i p}^{C} \geq Q_{i p}^{A V}$ in the upper branch.

\subsubsection{Choice of master node to evaluate}

As can be seen in the results section, Section 6, the number of B\&P nodes is small and we are able to explore the full tree. We therefore choose best first search for node selection to minimize the number of B\&P nodes solved. Another advantage of this compared to depth first search is that it generates a more diverse set of modes early on in the search and this is likely to improve the integer solutions found by RMP-IP.

When there is a choice between two child nodes of the same parent we choose the lower branch. This is likely to produce a mode with lower resource use than the upper branch and a mode with less resource use is more likely to generate a feasible solution in combination with other existing modes. This new solution may allow the other twin branch to be eliminated. 


\subsection{Branch \& Price algorithm}

Algorithm 1 shows a pseudo code of the basic version of the $\mathrm{B} \& \mathrm{P}$ algorithm we are using to solve the RTPO problem.

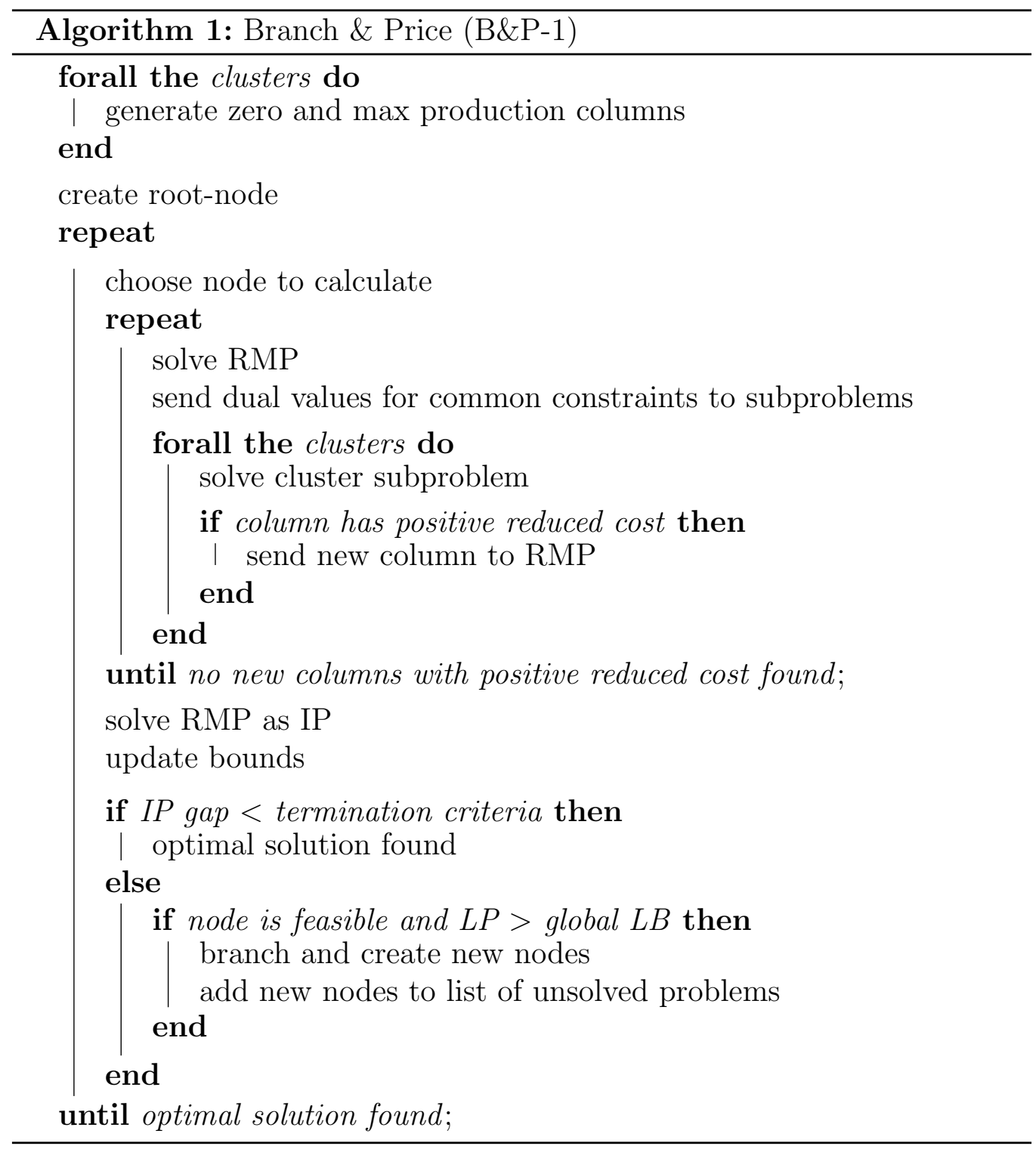

The B\&P algorithm starts by generating several initial columns i.e. modes of operating the clusters. This is done by creating a zero production column 
and a maximum production column for each cluster. This guarantees that there is an initial feasible solution to the continuous RMP at the root B\&P node. The next step is to solve the continuous RMP to find dual values on the common capacity constraints. These prices are sent to the subproblems, and all subproblems are solved. Each subproblem which returns with a positive reduced cost has its optimal solution added as a column to the RMP. The column generation procedure is repeated until no new positive reduced cost column is generated, at which stage the master problem at that node has been solved.

At this stage, the RMP-IP is solved to provide a feasible integer solution, and hence a lower bound on the optimal for the problem. If the gap between the upper bound and this feasible solution is within the predefined termination criteria, the algorithm terminates. Otherwise the B\&P algorithm creates two new problems based on the branching rules described in Section 4.6. These two problems are then added to the list of unsolved problems. The next step is to choose one of these unsolved problems based on the search strategy in Section 4.6.1, gather the set $\mathcal{S}_{i}$ of existing modes for cluster $i$ which satisfy the cluster's gas and water production limits, and run the column generation procedure over again.

We shall compare the following variants of the B\&P method

- B\&P-1 is our basic B\&P method, B\&P-2 and B\&P-3 builds on this method. It uses a best first search and branches on the gas or water production from clusters with fractional solution in the RMP, as described in Section 4.6. All subproblems are solved before the RMP is resolved. Each mode which has a positive reduced cost and is the most positive in its subproblem is added to RMP. To create feasible solutions the RMP-IP is solved using all the columns so far generated. This is done once per B\&P node after its column generation has converged.

- B\&P-2 is the same as B\&P-1 with 2 changes. Firstly the RMP is re-solved after each subproblem if a new production mode is found. Secondly when a subproblem fails to produce a column in one iteration it is not solved again until after an iteration which produces no columns. At that stage all the subproblems are again made active and the solution continues using the current dual prices.

- $\mathbf{B} \& \mathbf{P}-\mathbf{3}$ is the same as B\&P-2 except that after each iteration a three step procedure is used to generate a better incumbent. Firstly all sub- 
problems are solved with dual variables equal zero, and upper bounds on gas and water production equal to their RMP-IP solution plus the gap between their RMP-IP solution and the total production capacities (the slack on the two global constraints). Secondly, any of these columns that are better than the column for that subproblem selected in the RMP-IP are added to the RMP. Thirdly if any better columns have been found, the RMP-IP is solved again (which will give a better incumbent).

\section{Implementation}

The data for the piecewise linearization of the well and pipe models can be generated using any suitable simulators. In this paper we have used three state of the art simulators, GORM, [Mjaavatten et al., 2006] (an in house Statoil simulator) in combination with PROSPER, [Petroleum Experts, 2005], for the wells, and GAP, from Petroleum Experts [Petroleum Experts, 2010], for the pipes. These simulators are used currently in Troll operations. For each well we calculate the gas, oil and water flow for every wellhead pressure, and for each pipe we calculate the pressure drop through the pipe for every combination of gas, oil and water flow at the reference outlet pressure and temperature and estimate the gradient of pressure drop with outlet pressure. The results are then stored in tables in a form suitable for Xpress

In the Troll field the parallel pipelines are identical. In such situations swapping the set of wells attached to each pipeline produces an equivalent solution, and this introduces a symmetry which makes branch and bound for each subproblem much harder. We remove this symmetry, by selecting one well in each cluster that is likely to be producing and only allowing it to be routed to (at most) one of the pipelines.

The B\&P optimization algorithm is implemented in Mosel in Xpress-IVE [FICO, 2009] without any parallelization. "mmjobs" is used to jump between the sub and master problems, and breakpoint tables together with other topology information are included through data files. The computations were performed on single core of a Intel E5472, 3.0 GHz, 16GB RAM. Both Linux and Xpress were 64 bit versions. The solution times presented do not include time used to simulate well and pipe data. New runs for some of the well models i.e. for the wells that have changed behavior, is done before every optimization run, which in total takes typically a few seconds to a minute. 
The pipe models don't change so they need to be run only once.

\section{Results and discussion}

To validate our $\mathrm{B} \& \mathrm{P}$ method we have constructed a realistic model based on the structure of Troll B and C and we are using pipe and well data from these platforms. The structure was shown in Figure 1 and its size is comparable to the largest subsea production systems in the world. The full model has 8 clusters each containing 8 wells, 2 manifolds and 2 parallel pipelines. We wanted to compare our B\&P methods with solving this problem without any decomposition, however this full problem is too difficult to solve as a single MILP. To be able to do some comparisons, we have generated 9 other problems by choosing 2, 4 or 6 clusters from the full problem and reducing the common capacities constraints for gas and water to make them binding.

Section 2.1 compared the B\&P approach in this paper to the methods used in [Foss et al., 2009], [Gunnerud and Foss, 2010], [Gunnerud et al., 2010] and [Torgnes et al., 2012]. These papers tackle a problem with the same structure as the one in this paper. There are three major differences between these papers and this paper that affect the computational results. Firstly our B\&P methods will always find an optimal solution within a predefined tolerance level if they are allowed to run until termination, while the methods in the other papers cannot guarantee to achieve a predefined tolerance level. The tolerance of $0.01 \%$ used for all the B\&P runs reported in this paper is much smaller that is usually achieved by the methods in the other papers. Secondly we improve the accuracy of the model by including a term in the pressure drop equation (14) to allow for the effect of pipe outlet pressure. Thirdly we did new simulator runs to get more accurate representations of the pipeline models.

The purpose of the numerical study is to compare the four solution methods, Standard, i.e. the problem is solved as one large MILP without decomposition, B\&P-1, B\&P-2 and B\&P-3. Our three B\&P methods solved all our test cases to our predefined relative optimality tolerance of $0.01 \%$, so this has been omitted from the results tables.

The first two rows of the four result tables give the achieved relative optimality gaps and solution times for the Standard method. A dagger in the time field indicates that the run failed at that time because of lack of memory - on a 64 bit machine with 16 GB of RAM. After the first two rows 
there are three sets each of 3 rows. The first set shows the total run time for our $\mathrm{B} \& \mathrm{P}$ methods, the second set shows the number of $\mathrm{B} \& \mathrm{P}$ nodes, and the last set gives information on the number of subproblem solves. The first number in a cell is the number of subproblems solved and the second is the number of subproblems which did not generate a new production mode for the cluster.

Table 6: Results for 2 clusters

\begin{tabular}{|l|r|r|r|}
\multicolumn{1}{l}{ Problem } & \multicolumn{2}{c}{$2 \mathrm{~b}$} & \multicolumn{1}{c}{ c } \\
\hline Standard gap & $<0.01 \%$ & $<0.01 \%$ & $<0.01 \%$ \\
Standard time [min] & 0.7 & 0.5 & 39.7 \\
\hline B\&P-1 time [min] & 6.4 & 11.1 & 30.8 \\
B\&P-2 time [min] & 1.4 & 8.3 & 19.2 \\
B\&P-3 time [min] & 0.7 & 8.8 & 18.2 \\
\hline B\&P-1 nodes & 4 & 12 & 8 \\
B\&P-2 nodes & 4 & 11 & 8 \\
B\&P-3 nodes & 1 & 10 & 7 \\
\hline B\&P-1 subs s, & 24,14 & 60,36 & 64,31 \\
B\&P-2 subs s,z & 21,11 & 50,30 & 54,23 \\
B\&P-3 subs s,z & 15,6 & 55,30 & 49,18 \\
\hline
\end{tabular}

Table 6 shows the results for the three problems with 2 clusters. All the three problems were solved to optimality with the standard method, with a time that varied from under 1 to nearly 40 minutes. The time for the $\mathrm{B} \& \mathrm{P}$ methods varied in a similar range. The difference between $\mathrm{B} \& \mathrm{P}-2$ and $\mathrm{B} \& \mathrm{P}-$ 3 is small and both worked better than $\mathrm{B} \& \mathrm{P}-1$ for all the 3 test case. The number of subproblems that failed to yeild a new operating mode decreases from $\mathrm{B} \& \mathrm{P}-1$ to $\mathrm{B} \& \mathrm{P}-2$ and from $\mathrm{B} \& \mathrm{P}-2$ to $\mathrm{B} \& \mathrm{P}-3$.

Table 7 shows the results for the three test cases with problems with 4 clusters. The standard method only managed to solve one of the problems to optimality. All the B\&P methods solved all the problems to optimality in times significantly better than the times for the Standard method. The problem that was solved to optimality by the standard method took 153 minutes compared to between 26 and 52 minutes by the B\&P methods.

Table 8 shows the results for the three test cases with problems with 6 clusters. None of these problems were solved to optimality by the standard 
Table 7: Results for 4 clusters

\begin{tabular}{|l|r|r|r|}
\multicolumn{1}{l}{ Problem } & \multicolumn{1}{c}{$4 \mathrm{a}$} & \multicolumn{1}{c}{$4 \mathrm{~b}$} & \multicolumn{1}{c}{$4 \mathrm{c}$} \\
\hline Standard gap & $3.17 \%$ & $<0.01 \%$ & $4.01 \%$ \\
Standard time [min] & $181 \dagger$ & 153 & $174 \dagger$ \\
\hline B\&P-1 time [min] & 123 & 52 & 84 \\
B\&P-2 time [min] & 119 & 26 & 54 \\
B\&P-3 time [min] & 102 & 27 & 89 \\
\hline B\&P-1 nodes & 22 & 9 & 6 \\
B\&P-2 nodes & 22 & 9 & 6 \\
B\&P-3 nodes & 16 & 7 & 5 \\
\hline B\&P-1 subs s,Z & 192,149 & 120,79 & 64,42 \\
B\&P-2 subs s,Z & 175,134 & 95,58 & 60,41 \\
B\&P-3 subs s,z & 141,97 & 87,45 & 68,34 \\
\hline
\end{tabular}

Table 8: Results for 6 clusters

\begin{tabular}{|l|r|r|r|}
\multicolumn{1}{l}{ Problem } & \multicolumn{1}{c}{$6 \mathrm{a}$} & \multicolumn{1}{c}{$6 \mathrm{~b}$} & \multicolumn{1}{c}{$6 \mathrm{c}$} \\
\hline Standard gap & $9.51 \%$ & $6.99 \%$ & $11.3 \%$ \\
Standard time [min] & $430 \dagger$ & $519 \dagger$ & $394 \dagger$ \\
\hline B\&P-1 time [min] & 254 & 25 & 230 \\
B\&P-2 time [min] & 181 & 27 & 142 \\
B\&P-3 time [min] & 192 & 18 & 46 \\
\hline B\&P-1 nodes & 14 & 4 & 20 \\
B\&P-2 nodes & 13 & 4 & 19 \\
B\&P-3 nodes & 13 & 4 & 5 \\
\hline B\&P-1 subs s,z & 198,158 & 72,46 & 306,250 \\
B\&P-2 subs s,z & 152,121 & 68,46 & 240,189 \\
B\&P-3 subs s,z & 173,119 & 71,41 & 100,53 \\
\hline
\end{tabular}


method. The B\&P results for the 4 and the 6 cluster problems show similar variations between the test cases as we found for the 2 cluster problems.

Table 9: Average for 2, 4 and 6 clusters and Results for 8 clusters

Problem
\begin{tabular}{|l|r|r|r|r|}
\hline Standard gap & $<0.01 \%$ & $2.39 \%$ & $9.3 \%$ & $10.9 \%$ \\
Standard time [min] & 13.6 & $169 \dagger$ & $448 \dagger$ & $623 \dagger$ \\
\hline B\&P-1 time [min] & 16.1 & 86 & 170 & 195 \\
B\&P-2 time [min] & 9.6 & 66 & 117 & 125 \\
B\&P-3 time [min] & 9.3 & 73 & 85 & 121 \\
\hline B\&P-1 nodes & 8 & 12 & 13 & 14 \\
B\&P-2 nodes & 8 & 12 & 12 & 14 \\
B\&P-3 nodes & 6 & 9 & 7 & 13 \\
\hline B\&P-1 subs s,z & 49,27 & 125,90 & 192,151 & 264,213 \\
B\&P-2 subs s,z & 42,22 & 110,78 & 153,119 & 214,177 \\
B\&P-3 subs s,z & 40,18 & 99,59 & 115,71 & 245,172 \\
\hline
\end{tabular}

Table 9 compares our single test case with 8 clusters with the average of the results for the sets of problems with 2, 4 and 6 clusters. This table shows an increase in the relative gap for the standard method with increase in the number of clusters (though the highest relative gap for a single case is $11.3 \%$ for test case $6 \mathrm{c}$ ). The table also shows that both B\&P-2 and B\&P-3 are faster than $\mathrm{B} \& \mathrm{P}-1$ and that neither of the 2 faster ones dominates the other. The solution times for the $\mathrm{B} \& \mathrm{P}$ methods show very modest growth as the number of clusters in the problem increases.

The Standard method achieved the target tolerance of $0.01 \%$ only for the 2 cluster cases and for one of the 4 cluster cases. In all other cases it ran out of memory and failed with an unacceptably high bound gap and took a longer time for this that B\&P-1 took to solve the problem. For 9 of the 10 problems $\mathrm{B} \& \mathrm{P}-2$ is significantly faster than $\mathrm{B} \& \mathrm{P}-1$. The number of $\mathrm{B} \& \mathrm{P}$ nodes are similar, however the recalculation of the master solution after each subproblem solve and postponing resolving any subproblem that did not give a new production mode during its last solve has succeeded in significantly reducing the number of subproblem solved. By in addition utilizing the spare capacity of the common constraints as done in $\mathrm{B} \& \mathrm{P}-3$ we see a further improvement in solution time for 6 of the 10 problems. B\&P-3 never took 
more B\&P nodes than B\&P-2 for any of our test cases and it always had fewer subproblems that failed to find a new operating mode.

The RMP solution time is in the order of 0.01 second, while the RMP-IP solution time is usually one magnitude higher, and both these times are negligible compared to the subproblem solution times. Most subproblems solve in 5 - 120 seconds with 2000 - 100000 branch \& bound nodes. However, on some occasions, the subproblem can be much harder to solve, taking several thousand seconds and more than a million branch \& bound nodes. Small variations in the number of these slow solves can have a big effect on the solution time. The computational effort involved in solving the subproblems is mainly due to the piecewise linearization required to model the nonlinear well and pipe models. The well flow piecewise linearization have 20 to 100 breakpoints, and the pipe pressure drop linearization each have 343 breakpoints, corresponding to 7 breakpoints in each of the gas, water and oil flow SOS2s. There are 2 binary variables and one SOS2 per well and three SOS2s per pipe. Because of nonconvexities a lot of branching occurs on the SOS2s.

The optimization methods presented in this paper are intended as a tool for production engineers at oil and gas fields like Troll. Being able to find solutions overnight is of significant value, and all test problems in this study were solved by B\&P-3 within 3.25 hours, so comfortably satisfying this criterion. There are however occasions when faster solution times would be useful, for example if a well or cluster has to be closed down suddenly due to a production problem. Fortunately good suboptimal solutions are usually found much earlier in the solution process. Figure 3 shows for the 8 cluster example the variation over time of the gaps between the upper and lower bounds. This shows that B\&P-3 produced very good solutions within an hour for our largest test problem.

\section{Conclusion}

This paper presents a method that solves a Real Time Production Optimization problem (RTPO) using column generation in a Branch \& Price framework. The contribution lies in continuous branching on the cluster gas and water production variables, and on the MILP formulation of the subproblems which enables us to use a commercial solver. In addition, we utilize the spare capacity of the common constraints, after solving the restricted master problem, as an IP to create better feasible solutions. In this way we improve 


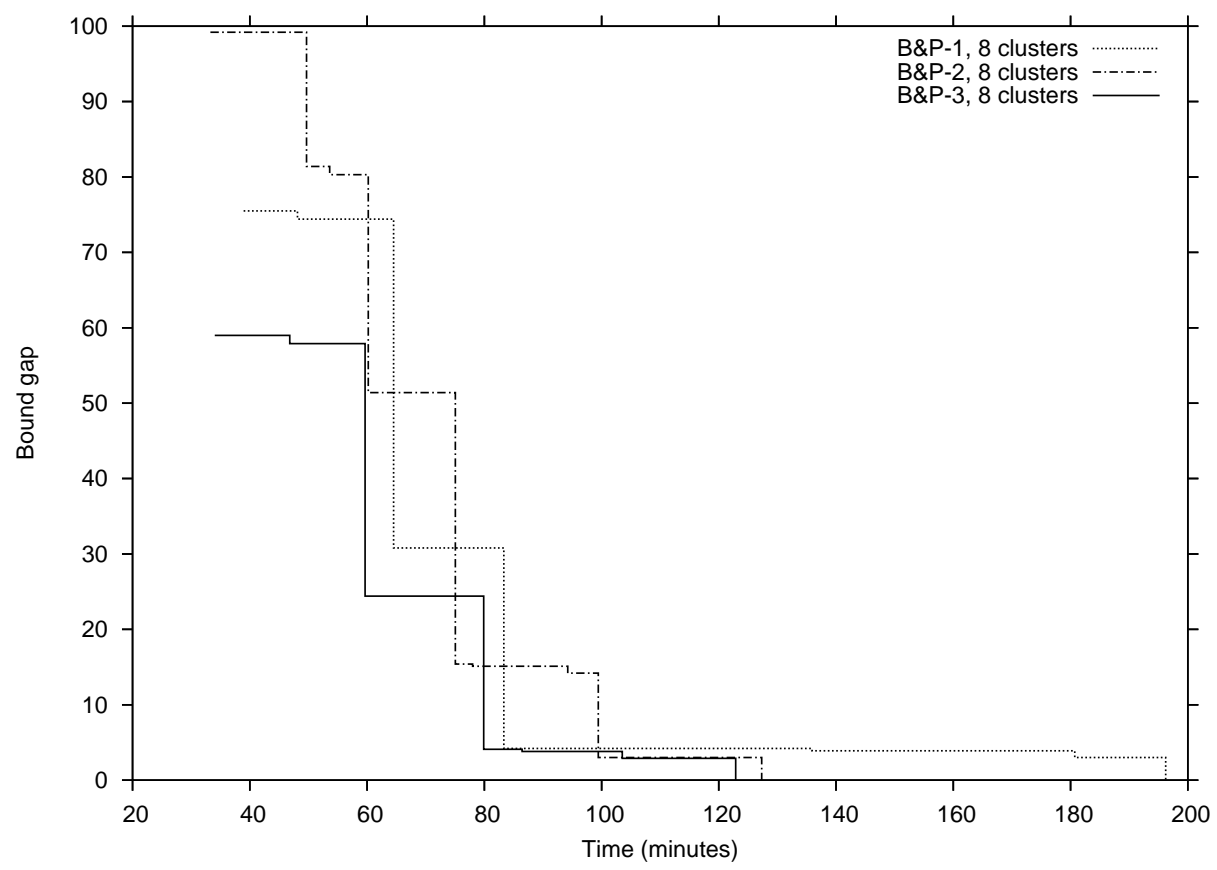

Figure 3: Bound gap as function of time for 8 cluster problem

the lower bound. Since the master problem solution time is negligible, it is re-solved each time a subproblems returns with a new column. To improve the solution time further, subproblems which once return without generating new columns are skipped until no further columns are generated, at which point the subproblems are solved again to establish the bounds.

The $\mathrm{B} \& \mathrm{P}$ method outperforms the standard implementation, which is only capable of finding the optimal solution of the smallest two cluster problems and one of the four cluster problems. The proposed algorithm terminates with no more than $22 \mathrm{~B} \& \mathrm{P}$ nodes for all tested cases, and near optimal solutions were always found in less than 1 hour.

In the Troll case there are 2 parallel pipelines in each cluster and pipelines join only at the separators. In some fields the clusters have different numbers of parallel pipelines and more complex topologies. Our model deals with an arbitrary number of parallel pipelines, however if modified slightly, it could deal with other topologies as well. The computational performance is unlikely to be affected by this change. For some fields a useful extension would be to include the optimization of gas lift or pump operation within the model. The same solution framework extends to this problem but with more linking constraints between the clusters. 
The RTPO problem is a challenge for the production engineers at Troll and other gas and oil producing installations with rate dependent gas, oil and water flow-fractions. The study performed in this paper was initiated by industrial partners, and has shown the potential to have significant impact when successfully integrated into their operating practices

\section{Acknowledgements}

We acknowledge the support of the Center for Integrated Operations at NTNU, Norway.

\section{References}

[Amr and de Carvalho, 2005] Amr, H. B. and de Carvalho, J. V. (2005). Cutting stock problems. In Desaulniers, G., Desrosiers, J., and Solomon, M. M. E., editors, Column Generation. Springer Science + Business Media, New York, USA.

[Barnhart et al., 1998] Barnhart, C., Johnson, E. L., Nemhauser, G. L., Savelsbergh, M. W. P., and Vance, P. H. (1998). Branch-and-price: Column generation for solving huge integer programs. Operation Research, 46 (3):316-332.

[Bieker, 2007] Bieker, H. (2007). Topics in offshore oil production optimization using real-time data. Ph. D. Dissertation, 200\%:148, Norwegian University of Science and Technology.

[Bieker et al., 2006] Bieker, H., Slupphaug, O., and Johansen, T. (2006). Real-time production optimization of offshore oil and gas production systems: A technology survey. SPE 99446, Journal SPE Production 83 Operations, 22:382-391.

[Desrosiers and Lübbecke, 2005] Desrosiers, J. and Lübbecke, M. E. (2005). A primer in column generation. In Desaulniers, G., Desrosiers, J., and Solomon, M. M. E., editors, Column Generation. Springer Science + Business Media, New York, USA.

[FICO, 2009] FICO (2009). Xpress-Mosel User guide. Release 3. 
[FMC Technologies, 2010] FMC Technologies, . (2010). http://www.fmctechnologies.com/22.10.2010.

[Foss et al., 2009] Foss, B., Gunnerud, V., and Dueñas Díez, M. (2009). Lagrangian decomposition of oil-production optimization applied to the troll west oil rim. SPE Journal SPE-118299-PA, Dec.

[Gunnerud and Foss, 2010] Gunnerud, V. and Foss, B. (2010). Oil production optimization - a piecewise linear model, solved with two decomposition strategies. Computers and Chemical Engineering, 34:1803-1812.

[Gunnerud et al., 2010] Gunnerud, V., Foss, B., and Torgnes, E. (2010). Parallel dantzig-wolfe decomposition for real-time optimization - applied to a complex oil field. Journal of Process Control, 20:1019-1026.

[Irnish and Desaulniers, 2005] Irnish, S. and Desaulniers, G. (2005). Shortest path problems with resource constraints. In Desaulniers, G., Desrosiers, J., and Solomon, M. M. E., editors, Column Generation. Springer Science + Business Media, New York, USA.

[Kosmidis et al., 2005] Kosmidis, V. D., Perkins, J. D., and Pistikopoulos, E. N. (2005). A mixed integer optimization for the well scheduling problem on petroleum fields. Computers and Chemical Engineering, 29:1523-1541.

[Mjaavatten et al., 2006] Mjaavatten, A., Aasheim, R., Saelid, S., and Gronning, O. (2006). A model for gas coning and rate-dependent gas/oil ratio in an oil-rim reservoir. Society of Petroleum Engineers, SPE 102390.

[Nygreen et al., 1998] Nygreen, B., Christiansen, M., Haugen, K., Bjorkvoll, T., and Kristiansend (1998). Modeling Norwegian petroleum production and transportation. Annals of Operations Research, 82:251 267.

[Petroleum Experts, 2005] Petroleum Experts, . (2005). User Guide PROSPER, Single Well Systems Analysis, version 9.1.

[Petroleum Experts, 2010] Petroleum Experts, . (2010). http://www.petex.com/22.10.2010.

[Saputelli et al., 2003] Saputelli, L., Mochizuki, S., Hutchins, L., Cramer, R., Anderson, M., and Muller, J. (2003). Promoting real-time optimization of hydrocarbon production systems. SPE 83978, Offshore Europe, 2-5 September 2003, Aberdeen, United Kingdom. 
[Torgnes et al., 2012] Torgnes, E., Gunnerud, V., Hagem, E., Rönnqvist, M., and Foss, B. (2012). Parallel Dantzig-Wolfe decomposition of petroleum production allocation problems. Journal of the Operational Research Society, page doi:10.1057/jors.2011.62.

[Wang, 2003] Wang, P. (2003). Development and applications of production optimization techniques for petroleum fields. Ph. D. Dissertation, Standford University.

[Weatherford, 2011] Weatherford, . (2011). http://www.weatherford.com /Products/Production /ProductionOptimization /Software/ 2.11.2011.

[Williams, 2005] Williams, H. P. (2005). Model Building in Mathematical Programming. Wiley. 